\title{
Correction
}

\section{Correction: Compan et al., How Does the Brain Implement Adaptive Decision Making to Eat?}

In the article "How Does the Brain Implement Adaptive Decision Making to Eat?" by Valérie Compan, B. Timothy Walsh, Walter Kaye, and Allan Geliebter, which appeared on pages 13868-13878 of the October 14, 2015 issue, two errors were found and are corrected below. These errors do not affect any of the conclusions or interpretations in the paper.

1. In Figure 4, the legend on the $x$-axis is missing. The corrected figure is shown below.
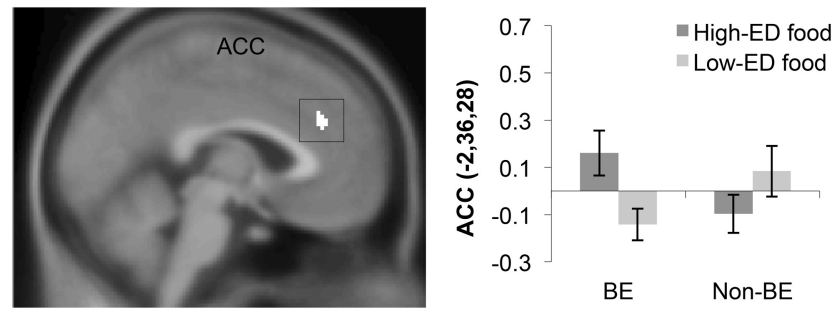

Figure 4.

2. On p. 13868, left column, the second sentence of the Introduction should be corrected as follows: Feeding behavior maintains the body weight to a threshold specific to one organism, and not to all organisms of one's species (as for temperature; Kupfermann, 1991).

DOI: 10.1523/JNEUROSCI.0264-16.2016 